\title{
Mini-Review: practice organization beyond memory processes
}

\section{GUILHERME M. LAGE¹ | LIDIANE A. FERNANDES² | TÉRCIO APOLINÁRIO-SOUZA³ | NATHÁLYA G. H. M. NOGUEIRA 1 | BÁRBARA P. FERREIRA ${ }^{1}$}

1 Universidade Federal de Minas Gerais - UFMG, Belo Horizonte, MG, Brasil.

2 Universidade Federal de Juiz de Fora - Campus Avançado de Governador Valadares - UFJF-GV, Governador Valadares, MG, Brasil.

${ }_{3}^{3}$ Instituto Metodista Izabela Hendrix, Belo Horizonte, MG, Brasil.

Correspondence to: Guilherme Menezes Lage, Department of Physical Education, Universidade Federal de Minas Gerais, Av. Pres. Antônio Carlos, 6627 Campus Pampulha, Belo Horizonte, CEP 31270-901, Brasil.

email: menezeslage@gmail.com

https://doi.org/10.20338/bjmb.v15i5.259

HIGHLIGHTS

- The second half of 2010 was a watershed in the history of practice organizations.

- Perceptual differences between more and less repetitive practices were found.

- Less repetitive practice (e.g., random) induces higher demand for processing.

- Parietal and occipital areas are more requested in less repetitive practice.

- Different neurophysiological measures have been applied.

\section{ABBREVIATIONS}

KR Knowledge of results

MT/N5+ Middle temporal region

PPC Posterior parietal cortex

\section{ABSTRACT}

Background: The benefits of variable practice in motor learning have been traditionally explained by the increased demand for memory processes induced by trial-to-trial changes. Recently, a new perspective associating increased demand for perception with variable practice has emerged.

Aim: This revision aims to present and discuss the findings in this exciting topic newly opened.

Results / Interpretation: In the second half of 2010's, a number of studies have pointed out differences in perceptual processing when compared variable and repetitive practices. Different levels of (a) hemodynamic activation, (b) electroencephalographic activity, (c) neurochemical activity, and (d) oculomotor behavior have provided evidence that perceptual processes are affected differently by variable and repetitive practices.

KEYWORDS: Practice organization | Memory | Perceptual mechanisms | Mental workload

\section{INTRODUCTION}

This review aims to synthesize and update the recent findings of an important topic investigated in the Motor Learning area, the practice organization. These findings have the potential to redirect the research efforts in this field. To achieve such an aim, the review was structured into two main sections. The "focus on memory" section presents to the reader the schedules of practice and the traditional behavioral hypotheses that explain the benefits of the more variable practices, compared with more repetitive ones, through memory processes. Then, the "focus on perception" section aims to explore new explanations beyond memory processes that have recently been presented in the literature. The new findings involving perceptual functions and brain areas are presented in chronological order. These discoveries expand our knowledge about the mechanisms that underlie the practice organization. 


\section{PRACTICE ORGANIZATION: THE FOCUS ON MEMORY PROCESSES}

Two important factors that influence the quality and quantity of information received, processed, and generated by the learner are the number and order of skills. ${ }^{1}$ In this sense, different levels of processing are expected when comparing more repetitive and more variable types of practice organizations. In essence, there are two practice schedules: constant and variable. ${ }^{2}$ The constant practice consists of learning only one skill during a practice session. Thus, only a repetitive order of trials is possible. Variable practice refers to learning two or more skills, ${ }^{3}$ as consequence, different orders of skills presentation are possible (Figure 1a).

Practices that are more variable lead to better learning than more repetitive practices. While variable practices result in more effective motor learning than constant practice, ${ }^{4}$ random and serial variable practices are more effective than the blocked variable practice. $5,6 \mathrm{~A}$ point of convergence in all proposed behavioral hypotheses is that more variable practices demand more from mnemonic processes than more repetitive practices, being this feature responsible for the better learning observed in random and serial practices when compared with constant and blocked practices.

The variability of practice hypothesis claims that variable practices produce stronger memory schemas than constant practice. 7,8 The elaborative-processing hypothesis proposes that random practice, compared with the blocked practice, leads to increased elaboration and distinction of skills in memory. ${ }^{9}$ Lastly, the forgettingreconstruction hypothesis suggests a stronger involvement of random practice in processes of forgetting and reconstruction of action plans of the skills in memory. ${ }^{10}$ Altogether, these behavioral hypotheses directed the efforts of investigation about practice organization for more than 40 years from the behavioral level of analysis ${ }^{11}$ to the molecular level of analysis. ${ }^{12}$

These efforts resulted in a significant advance in our knowledge about processes involving working memory and long-term memory. As well as, we improved deeply our view about neurobiological mechanisms involved in more repetitive and variable practices. For example, during movement preparation, more variable practice promotes increased activity in the premotor cortex and the supplementary motor area throughout the practice.13,14 Regarding the movement execution phase, increased levels of activity in the supplementary motor area, ${ }^{13}$ premotor cortex, and primary cortex ${ }^{14}$ were observed during random practice over the course of practice. Altogether, these results show that the cortical structures from the frontal lobe, involved in the planning and execution of skills, exhibit greater activity during random practice than during blocked practice, ${ }^{2}$ as exhibited in Figure $1 \mathrm{~b}$. 


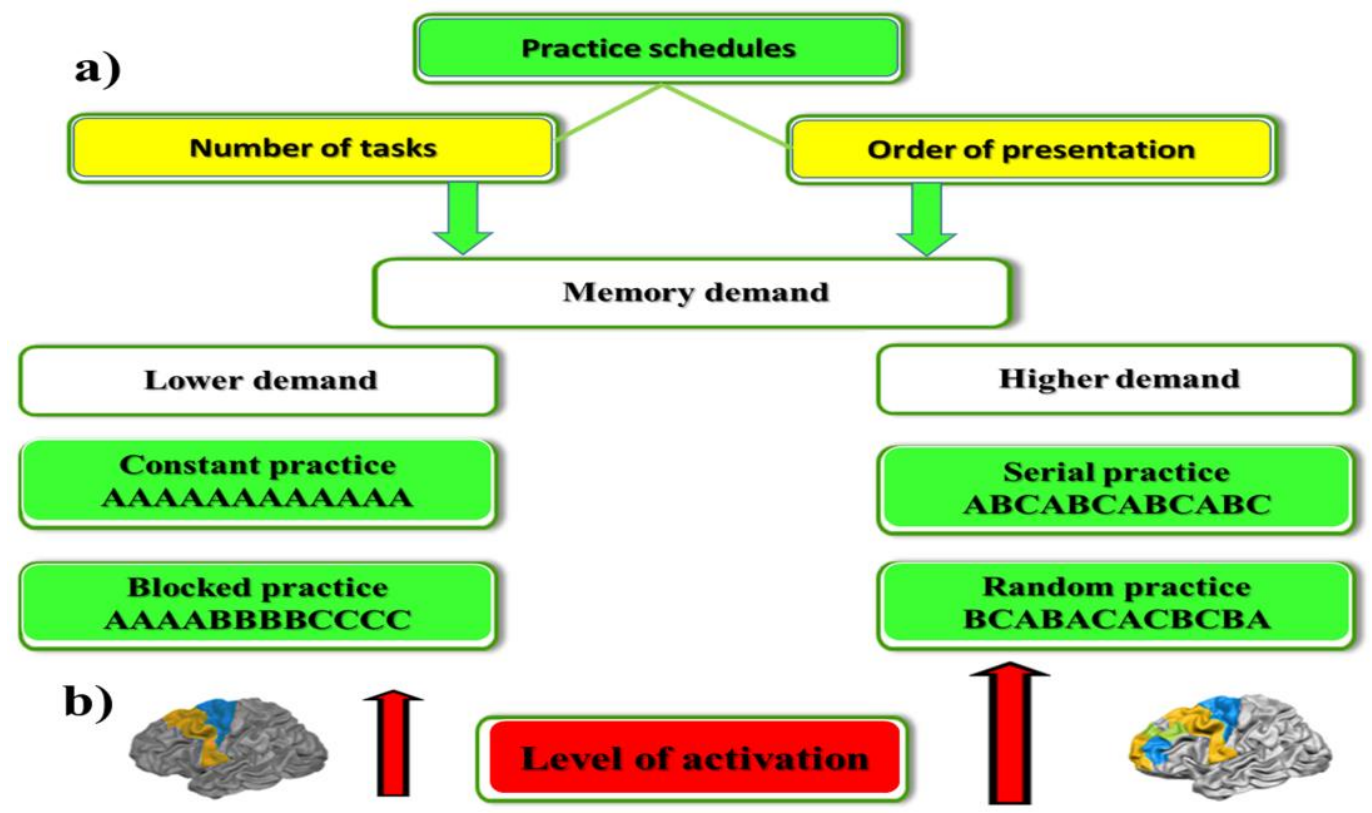

Figure 1. The number and order of skills impact on memory processes. (a) Serial and random practices present higher demand on memory, as well as (b) these practices present increased activity of frontal areas of the brain.

\section{PRACTICE ORGANIZATION: COULD PERCEPTUAL PROCESSES BE AFFECTED?}

From the very firsts behavioral studies in 19797,8 until the first two reviews in 2015 and 2016 regarding neurobiological aspects involved in practice organization,,215 much less has been discussed beyond the effects on memory processes. From a sensorimotor perspective, much has been studied about the motor output and almost nothing has been investigated in terms of sensory input. Only in 2017, two studies addressed the role of perceptual aspects in practice organization. ${ }^{16,17}$ Lelis-Torres et al. ${ }^{16}$ investigated the level of mental workload involved in random and constant practice with an electrophysiological approach. They applied two electroencephalography measures related to cognitive states. A former involving inference about working memory load, and a second concerning sensory processing and attention resources involved in information-gathering and visual scanning. The results showed that random practice induced a higher mental workload, compared with constant practice, in terms of perceptual processing. For the first time, it was observed a higher level of task engagement reflecting the allocation of perceptual processing associated with visual scanning and information gathering in practice that is more variable.

The results of Lelis-Torres et al. ${ }^{16}$ were interpreted through the necessity of dealing with continuous changes imposed trial-by-trial in random practice. In each new trial, the learned needs to perform visual scanning to gather information about the new goal for the next trial. Conversely, the relevance of visual scanning to gather information to movement planning should be lower, taking into account the trial-to-trial stability promoted by the consecutive repetition of the goal task in constant practice. There is no need for continuous visual search since the learner can maintain the goal task active in working 
memory. Another point discussed by the authors was the role of visual scanning and gathering information on the feedback information. Once the trial execution is finished, two main sources of feedback information are usually available for the learner. The former is the information about intrinsic feedback, and the second is the extrinsic information supplied by knowledge of results (KR). In both constant and random conditions of practice, the learner needs to gather these types of information to feed the next action planning. However, differences in time or frequency of visual fixation spent in KR information could be expected because the levels of errors differ between variable and repetitive practices. Bicalho et al. ${ }^{18}$ posteriorly investigated this hypothesis.

Another study investigating perceptual processing in practice organization was also published in 2017. Thürer et al. ${ }^{17}$ observed that mechanisms related to online feedback are increased within the parietal cortex in random practice, compared with blocked practice. The parietal cortex is the main control center for sensory feedback in the brain. More specifically, the posterior parietal cortex (PPC) stands out for its importance in the formation of sensory representations that are involved in both movement planning and error corrections. ${ }^{19}$ In motor adaptation tasks the unpredictability of perturbations under random practice, forces the learner to correct their movements during the execution requiring more from perceptual processes, and consequently, from brains areas involved in processing online feedback. ${ }^{17}$

Surprisingly, after these two studies in 2017, three new studies investigating perceptual processes in practice organization were published in 2018.20,21,22 Pauwels et al.22 observed that random practice leads to higher recruitment of brain areas associated with the planning and execution of a bimanual visuomotor task. Increased intertask comparisons in random practice induced a higher level of activation of areas involved in visual processing. Pauwels and colleagues linked the behavioral hypothesis of elaborativeprocessing with neural substrates involved in visual processing, particularly the middle temporal region (MT/V5+). This brain region presents a central role in visual motion perception, ${ }^{23}$ involving, among other functions, feedback processing. ${ }^{24}$ Pauwels et al. ${ }^{22}$ also observed that the bilateral lateral occipital cortex, precuneus, and V1 remained more active in random practice compared to the blocked practice during the retention test. A possible explanation is that these visual processing areas became an intrinsic part of the motor representation, even when visual feedback is not supplied.

Chalavi et al. ${ }^{20}$ hypothesized that random practice would decrease more the levels of GABA, the main inhibitory neurotransmitter in the brain, than blocked practice. Motor learning modulates the GABAergic (gamma-aminobutyric acid) system within the sensorimotor cortex. This hypothesis was partially confirmed, since decreased GABA levels in random practice were found in the sensorimotor and occipital cortex. The bimanual task applied imposed the use of visual information processing during practice demanding not only activation of the sensorimotor cortex, but also the occipital cortex. An unexpected finding was the increased GABA level from pre-training from post-traininginduced by blocked practice. The explanation for this finding was based on the rationale that repeated presentation of stimuli results in reduced neuronal responses, a phenomenon defined as "repetition suppression". The repetitive context of task execution involved in blocked practice increases the GABA level within the occipital cortex in conjunction with a decrease of activity in the task-irrelevant neurons and an increase in the task-relevant neurons. This assumption needs to be investigated. 
Henz et al. ${ }^{21}$ conducted the third study published in 2018, in which different practice protocols were compared in the learning of badminton serves. Variable practice protocols involving random variations of tasks and differential learning (variation of movement solutions) were applied, as well as a repetitive practice. Distinct neural processes were found after these practice protocols. Variations of a movement pattern applied in differential learning protocol increased more electroencephalography theta and alpha power within somatosensory central and parietal regions than a random variation of tasks and repetitive practice. Henz et al. ${ }^{21}$ proposed that differential learning induces more integration from different sensory modalities compared to the random variation of tasks and repetitive practice protocols. Taking into account the theoretical difference of the differential learning approach in comparison with the other studies analyzed, ${ }^{16,20,22}$ the conjunction of findings show that variation of parameters of the same task induces different perceptual processing compared to the repetitive practice. Only Henz et al. ${ }^{21}$ applied a random variation of different tasks, and they did not find differences between variable and repetitive practices. More studies are necessary to clarify whether intra- and inter-task variations induce different levels of perceptual processing.

Bicalho et al. ${ }^{18}$ published the last article on this subject during the second half of The 2010's. They extended the knowledge about mental effort and perceptual differences provided by Lelis-Torres et al. ${ }^{16}$ in practice organization through oculomotor analyses. Lelis-Torres et al. ${ }^{16}$ hypothesized that visual scanning to gather information would be different from practice schedules. In sequential learning tasks with two temporal goals to be learned, information about the next goal to be planning and information about errors, or $\mathrm{KR}$, is supplied concerning the goals related to absolute timing, or the parameters of movement, and relative timing, or pattern of movement. The hypothesis proposes that learners in variable practice direct their attention more to the absolute dimension of the task because they are concerned about the trial-to-trial changes in the absolute goal. On the contrary, during repetitive practice, attention is focused more on the relative goal of the task, which is maintained fixed trial-to-trial. This proposition was based on behavioral findings, which indicated that constant practice benefits learning of the relative timing of the movement, and random practice benefits the learning of the absolute timing. ${ }^{4,25,26}$

The results found by Bicalho et al. ${ }^{18}$ confirmed partially the hypothesis. As expected, random practice showed a higher amount of KR and goal information gathered from the absolute dimension than constant practice. These differences were found in both KR period and planning period. However, constant practice, compared to the random practice, showed higher amounts of goal information gathered only from goal, but not from $\mathrm{KR}$. As random practice induces higher levels of errors in both absolute and relative goals, learners cannot direct their visual attention to only one dimension. Another hypothesis proposed by Lelis-Torres et al..$^{16}$ and investigated by Bicalho et al. ${ }^{18}$ was that, throughout practice, a constant practice should decrease more visual scanning because of its repetitive nature. The learner could maintain in working memory the information about goals. The results showed that both groups diminished the gathering of this information from the first to the last block of the acquisition phase but, random practice exhibited a higher amount of information gathering since the first block of trials. Taken together, the results indicate many differences in visual scanning associated with variable and repetitive practices. Additionally, pupil dilation and amount of eyeblinks were used as dependent variables to inform about mental workload involved in variable and repetitive practices. A 
higher level of pupil dilation and amount of eyeblinks were induced by variable practice compared to the repetitive practice. Figure 2 synthesizes all findings from Bicalho et al. ${ }^{18}$ and previous studies presented.

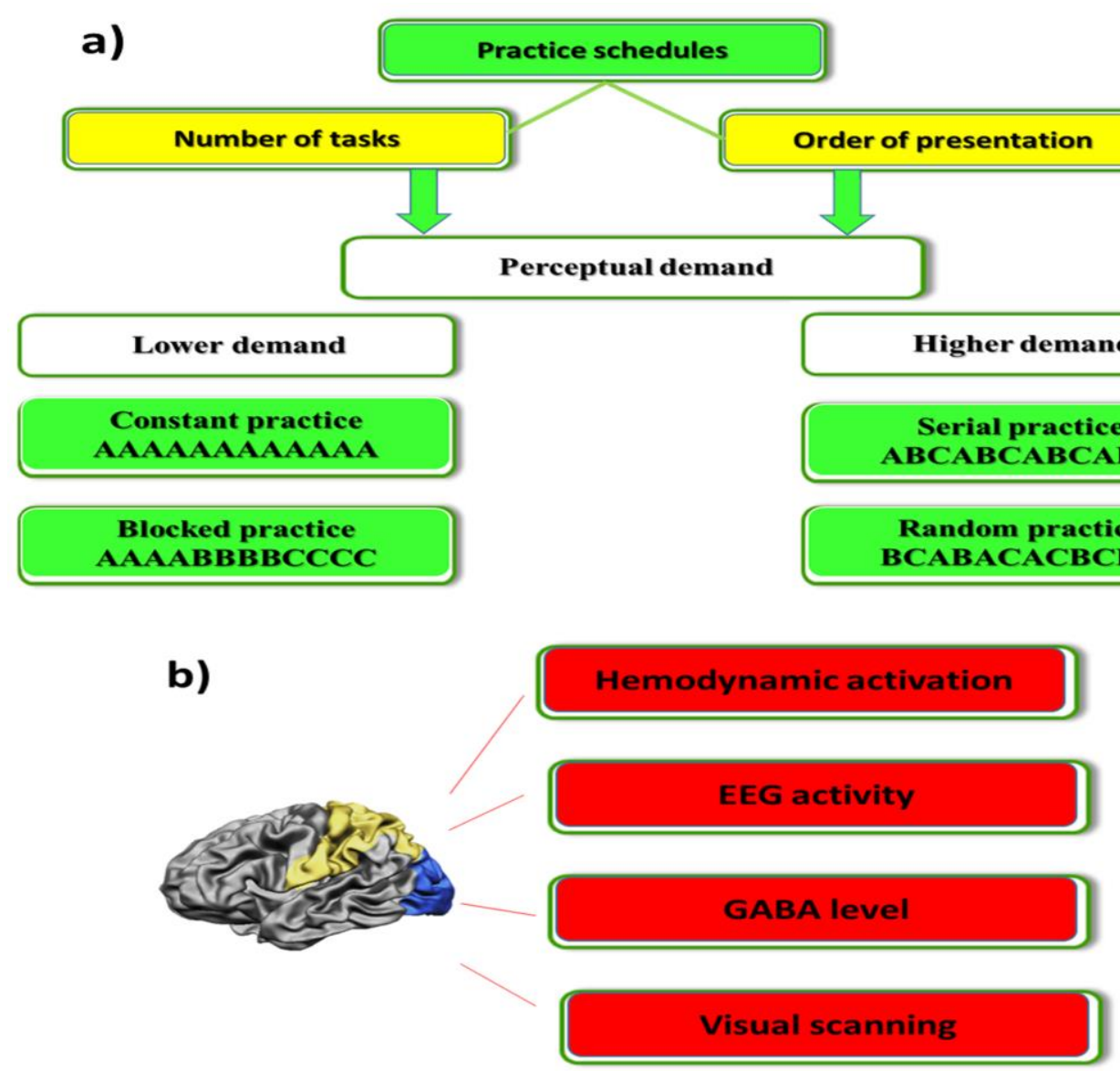

Figure 2. The number and order of skills impact on perceptual processes. (a) Serial and random practices present higher demand on perception. (b) Differences in parietal and occipital areas were found in different behavioral and neurobiological measures.

\section{CONCLUSION}

In conclusion, the second half of the 2010's can be considered a watershed in the practice organization history. Several studies pointed out news possibilities to thought perceptual mechanisms involved in more variable and repetitive practices. Some popular topics in the Motor Learning area have been revisited by neuroscientists, ${ }^{27}$ and the organization of practice has been profoundly beneficiated by this movement. As quoted by Lelis-Torres et al., 16 "the increased demand for sensory processing observed in random practice opens a new exciting field of study in practice organization".

\section{REFERENCES}

1. Gonçalves WR, Lage GM, Silva A, Ugrinowitsch H, Benda RN. O efeito da interferência contextual em idosos. Rev Port Cien Desp. 2007;7:217-224. doi: 10.5628/rpcd.07.02.217. 
2. Lage GM, Ugrinowitsch H, Apolinario-Souza T, Vieira MM, Albuquerque MR, Benda RN. Repetition and variation in motor practice: A review of neural correlates. Neurosci Biobehav Rev. 2015;57:132-141. doi: 10.1016/j.neubiorev.2015.08.012.

3. Shea CH, Kohl RM. Specificity and variability of practice. Res Q Exerc Sport. 1990;61:169177. doi: 10.1080/02701367.1990.10608671.

4. Lage GM, Apolinário-Souza T, Albuquerque MR, Portes LL, Januário MDS, Vieira MM, et al. The effect of constant practice in transfer tests. Motriz. 2017;23:22-32. doi: 10.1590/S1980-6574201700010004.

5. Sekiya H, Magill RA, Sidaway B, Anderson DI. The contextual interference effect for skill variations from the same and different generalized motor programs. Res Q Exerc Sport. 1994;65:330-338. doi: 10.1080/02701367.1994.10607637.

6. Thurer B, Gedemer S, Focke A, Stein T. Contextual Interference Effect Is Independent of Retroactive Inhibition but Variable Practice Is Not Always Beneficial. Front Hum Neurosci. 2019;13:165. doi: 10.3389/fnhum.2019.00165.

7. Moxley SE. Schema: the variability of practice hypothesis. J Mot Behav. 1979;11:65-70. doi: $10.1080 / 00222895.1979 .10735173$.

8. Shea JB, Morgan RL. Contextual interference effects on the acquisition, retention, and transfer of a motor skill. J Exp Psychol Hum Learn. 1979;5:179-187. doi: 10.1037/02787393.5.2.179.

9. Shea JB, Zimny ST. Context Effects in Memory and Learning Movement Information. In: Magill RA, editor. Advances in Psychology. North-Holland; 1983:pp.345-366.

10. Lee TD, Magill RA. The locus of contextual interference in motor-skill acquisition. J Exp Psychol: Learn Mem Cogn. 1983;9:730-746. doi: 10.1037/0278-7393.9.4.730.

11. Wright $\mathrm{DL}$. The role of intertask and intratask processing in acquisition and retention of motor skills. J Mot Behav. 1991;23:139-145. doi: 10.1080/00222895.1991.9942031.

12. Apolinario-Souza T, Santos Almeida AF, Lelis-Torres N, Otoni Parma J, Pereira GS, Menezes Lage G. Molecular Mechanisms Associated with the Benefits of Variable Practice in Motor Learning. J Mot Behav. 2020;52:515-526. doi: 10.1080/00222895.2019.1649997.

13. Cross ES, Schmitt PJ, Grafton ST. Neural substrates of contextual interference during motor learning support a model of active preparation. J Cogn Neurosci. 2007;19:18541871. doi: 10.1162/jocn.2007.19.11.1854.

14. Wymbs NF, Grafton ST. Neural Substrates of Practice Structure That Support Future OffLine Learning. J Neurophysiol. 2009;102:2462-2476. doi: 10.1152/jn.00315.2009

15. Wright D, Verwey W, Buchanen J, Chen J, Rhee J, Immink M. Consolidating behavioral and neurophysiologic findings to explain the influence of contextual interference during motor sequence learning. Psychon Bull Rev. 2016;23:1-21. doi: 10.3758/s13423-0150887-3

16. Lelis-Torres N, Ugrinowitsch H, Apolinario-Souza T, Benda RN, Lage GM. Task engagement and mental workload involved in variation and repetition of a motor skill. Sci Rep. 2017;7:14764. doi: 10.1038/s41598-017-15343-3. 
17. Thurer B, Stockinger C, Putze F, Schultz T, Stein T. Mechanisms within the Parietal Cortex Correlate with the Benefits of Random Practice in Motor Adaptation. Front Hum Neurosci. 2017;11:403. doi: 10.3389/fnhum.2017.00403.

18. Bicalho LEA, Albuquerque MR, Ugrinowitsch H, Da Costa VT, Parma JO, Dos Santos Ribeiro T, et al. Oculomotor behavior and the level of repetition in motor practice: Effects on pupil dilation, eyeblinks and visual scanning. Hum Mov Sci. 2019;64:142-152. doi: 10.1016/j.humov.2019.02.001.

19. Oliveira JRV, Romano-Silva MA, Ugrinowitsch H, Apolinario-Souza T, Fernandes LA, Parma JO, et al. Cathodal tDCS of the Left Posterior Parietal Cortex Increases Proprioceptive Drift. J Mot Behav. 2019;51:272-280. doi: 10.1080/00222895.2018.1468311.

20. Chalavi S, Pauwels L, Heise KF, Zivari Adab H, Maes C, Puts NaJ, et al. The neurochemical basis of the contextual interference effect. Neurobiol Aging. 2018;66:85-96. doi: 10.1016/j.neurobiolaging.2018.02.014.

21. Henz D, John A, Merz C, Schollhorn WI. Post-task Effects on EEG Brain Activity Differ for Various Differential Learning and Contextual Interference Protocols. Front Hum Neurosci. 2018;12:19. doi: 10.3389/fnhum.2018.00019.

22. Pauwels L, Chalavi S, Gooijers J, Maes C, Albouy G, Sunaert S, et al. Challenge to Promote Change: The Neural Basis of the Contextual Interference Effect in Young and Older Adults. J Neurosci. 2018;38:3333-3345. doi: 10.1523/JNEUROSCI.2640-17.2018.

23. Galletti C, Fattori $P$. The dorsal visual stream revisited: Stable circuits or dynamic pathways? Cortex; a journal devoted to the study of the nervous system and behavior. 2018;98:203-217. doi: 10.1016/j.cortex.2017.01.009.

24. Beets IA, Gooijers J, Boisgontier MP, Pauwels L, Coxon JP, Wittenberg G, et al. Reduced Neural Differentiation Between Feedback Conditions After Bimanual Coordination Training with and without Augmented Visual Feedback. Cereb Cortex. 2015;25:1958-1969. doi: 10.1093/cercor/bhu005.

25. Lai Q, Shea CH. Generalized Motor Program (GMP) Learning: Effects of Reduced Frequency of Knowledge of Results and Practice Variability. J Mot Behav. 1998;30:51-59. doi: 10.1080/00222899809601322.

26. Lai $\mathrm{Q}$, Shea $\mathrm{CH}$, Wulf $\mathrm{G}$, Wright $\mathrm{DL}$. Optimizing generalized motor program and parameter learning. Res Q Exerc Sport. 2000;71:10-24. doi: 10.1080/02701367.2000.10608876.

27. Lee TD, Carnahan H. Motor Learning: Reflections on the Past 40 Years of Research. Kinesiol Rev. 2021;10:274-282. doi: 10.1123/kr.2021-0018.

\section{ACKNOWLEDGMENTS}

We thank the Scientific Board of the Brazilian Society of Motor Behavior for the invitation. This edition celebrating the $15^{\text {th }}$ Anniversary of BJMB is very special. Long live to the BJMB. 
Citation: Lage GM, Fernandes LA, Apolinário-Souza T, Nogueira NGHM, Ferreira BP. (2021). Mini-Review: practice organization beyond memory processes. Brazilian Journal of Motor Behavior, 15(5):333-341.

Editors: Dr Fabio Augusto Barbieri - São Paulo State University (UNESP), Bauru, SP, Brazil; Dr José Angelo Barela São Paulo State University (UNESP), Rio Claro, SP, Brazil; Dr Natalia Madalena Rinaldi - Federal University of Espírito Santo (UFES), Vitória, ES, Brazil.

Copyright:@ 2021 Lage, Fernandes, Apolinário-Souza, Nogueira and Ferreira and BJMB. This is an open-access article distributed under the terms of the Creative Commons Attribution-Non Commercial-No Derivatives 4.0

International License which permits unrestricted use, distribution, and reproduction in any medium, provided the original author and source are credited.

Funding: This research did not receive any specific grant from funding agencies in the public, commercial, or not-forprofit sectors.

Competing interests: The authors have declared that no competing interests exist.

DOI: https://doi.org/10.20338/bjmb.v15i5.259 